OPEN

SUBJECT AREAS: ELECTROCATALYSIS

BATTERIES

Received

4 July 2014

Accepted

15 August 2014

Published

5 September 2014

Correspondence and requests for materials should be addressed to

Q.H.Y. lqhyangcn@ tju.edu.cn) or D.-W.W. (da-wei.wang@unsw. edu.au)

* These authors contributed equally to this work.

\section{Unusual High Oxygen Reduction Performance in All-Carbon} Electrocatalysts

\author{
Wei Weil ${ }^{14}$, Ying Tao ${ }^{1,4 *}$, Wei Lv²*, Fang-Yuan Su², Lei Ke² ${ }^{2}$ Jia Li² ${ }^{2}$ Da-Wei Wang ${ }^{3}$, Baohua Li², \\ Feiyu Kang ${ }^{2} \&$ Quan-Hong Yang ${ }^{1,2,4}$
}

'Key Laboratory for Green Chemical Technology of Ministry of Education, School of Chemical Engineering and Technology, Tianjin University, Tianjin, 300072, China, ${ }^{2}$ Engineering Laboratory for Functionalized Carbon Materials, Graduate School at Shenzhen, Tsinghua University, Shenzhen, 518055, China, ${ }^{3}$ School of Chemical Engineering, The University of New South Wales, Sydney, NSW 2033, Australia, ${ }^{4}$ The Synergistic Innovation Center of Chemistry and Chemical Engineering of Tianjin, Tianjin, 300072, China.

Carbon-based electrocatalysts are more durable and cost-effective than noble materials for the oxygen reduction reaction (ORR), which is an important process in energy conversion technologies. Heteroatoms are considered responsible for the excellent ORR performance in many carbon-based electrocatalysts. But whether an all-carbon electrocatalyst can effectively reduce oxygen is unknown. We subtly engineered the interfaces between planar graphene sheets and curved carbon nanotubes (G-CNT) and gained a remarkable activity/selectivity for ORR (larger current, and $n=3.86, \sim 93 \%$ hydroxide $+\sim 7 \%$ peroxide). This performance is close to that of Pt; and the durability is much better than Pt. We further demonstrate the application of this G-CNT hybrid as an all-carbon cathode catalyst for lithium oxygen batteries.We speculate that the high ORR activity of this G-CNT hybrid stems from the localized charge separation at the interface of the graphene and carbon nanotube, which results from the tunneling electron transfer due to the Fermi level mismatch on the planar and curved $s p^{2}$ surfaces. Our result represents a conceptual breakthrough and pioneers the new avenues towards practical all-carbon electrocatalysis.

igh-energy metal-air batteries are of paramount importance for the resolution of fossil fuel depletion and $\mathrm{CO}_{2}$ emission. These batteries are key parts for future renewable energy framework because of their extraordinary energy density, which is at least ten times larger than that of lithium-ion batteries ${ }^{1}$. Oxygen reduction reaction (ORR) on the air-breathing cathodes of metal-air batteries plays a determinant role in maximizing the overall device performance. Theoretically, the complete reduction of oxygen in aqueous electrolytes produces $\mathrm{OH}^{-}$(alkaline) or $\mathrm{H}_{2} \mathrm{O}$ (acidic), giving rise to an electron transfer number (n) of $4^{2,3}$. The state-of-the-art electrocatalyst for ORR is Pt. ORR catalyzed by Pt-based catalysts has demonstrated high onset potential and fast kinetics and is a four-electron transfer process. But Pt-based catalysts are often expensive and less resistant to dissolution, coalescence or poisoning ${ }^{4}$. Among many kinds of non-noble ORR catalysts ${ }^{3-8}$, heterogeneous carbon materials have shown increasing importance. Enormous studies elucidated the improved ORR activity in different kinds of doped carbon materials, such as carbon nanotubes (CNTs), graphene sheets, and nanoporous carbons ${ }^{9}$. These metal-free catalysts prevent the leaching or poisoning of metal atoms and hence render superb stability. The common dopants are nitrogen ${ }^{10-14}$, boron ${ }^{15}$, sulfur ${ }^{16}$, or sulfur/nitrogen co-dopant ${ }^{17}$. These dopants are usually covalently bonded with carbon atoms. Theoretical simulation speculated the mechanism of doping is to generate charge separation in the charge-neutral $s p^{2}$ carbon lattice, which facilitates oxygen adsorption $^{14}$.

Unlike the covalent doping (charge injection), we propose a non-covalent doping strategy to enhance the ORR activity of all-carbon electrocatalysts. The idea is to make use of the different electronic structures of carbon allotropes ${ }^{18,19}$. The adsorption of dioxygen molecules and the transfer of electrons are the rate determining steps for ORR catalyzed by carbon materials ${ }^{3}$. The $\pi$ electrons in a $s p^{2}$ carbon lattice distribute homogeneously; whereas doping-induced uneven charge distribution is the key to high ORR activity. Non-covalent doping of $s p^{2}$ carbon can be induced by the work function difference between metal and graphene ${ }^{20,21}$. For example, Au can attract electrons from graphene due to its larger work function, and hence apply a $p$-type doping to graphene ${ }^{22}$. The electron transfer from graphene to CNTs was also attributed to the larger work function of $\mathrm{CNTs}^{23,24}$. 
Theoretically speaking, non-covalent $p$-doping of graphene can be achieved by taking CNTs as electron acceptors ${ }^{25}$. The charge separation would naturally occur at the interfaces of CNT and graphene ${ }^{25}$.

In this communication, we successfully engineered the interface between CNTs and graphene sheets, and developed a CNT-doped graphene hybrid (G-CNT) for the use as an all-carbon ORR catalyst, free from metal or non-carbon dopants. Such an all-carbon catalyst developed a 3D hierarchical porous open network consisting of entangled graphene sheets and CNTs. Although sole carbon allotropes have limited ORR selectivity $(2<\mathrm{n}<3)^{8,26}$, we demonstrate the enhanced ORR selectivity $(n=3.86)$ and reaction current in our G-CNT hybrids where the interface structure is delicate. We show for the first time that all-carbon materials are electrocatalytically active and stable, and most importantly, more cost effective. We speculate that the high ORR activity of this G-CNT hybrids stems from the charge separation at the interface of the graphene and carbon nanotube, which results from the electron tunneling due to the Fermi level mismatch at the planar and curved $s p^{2}$ surfaces. Taking into account of the facilitated oxygen diffusion paths, and the excellent ORR activity, we evaluated the $3 \mathrm{D}$ hybrid in a Lithium- $\mathrm{O}_{2}$ cell which showed high performance.

\section{Results}

Fabrication and structure characterization of all-carbon catalysts. For the preparation of a typical G-CNT monolith, a homogeneously mixed aqueous suspension of graphene oxide (GO) and CNTs with the mass ratios of 100/20 was hydrothermally treated in a Teflonlined autoclave at $150^{\circ} \mathrm{C}$ for $20 \mathrm{~h}$ to form a hybrid hydrogel (Figure $\mathrm{S} 1$ ), which was then freeze-dried and annealed at $300^{\circ} \mathrm{C}$ for $2 \mathrm{~h}$ and $800^{\circ} \mathrm{C}$ for $3 \mathrm{~h}$ in Ar. A series of G-CNT hybrids were obtained following the protocol in Figure 1. These samples are denoted G-CNT $X / Y$, where $X=100$ (GO content) and $Y=20,10,5,3.3$, and 2 (CNT content). The details for the preparation are provided in the method section.

The microstructure and morphology of the as-prepared GCNT100/20 were investigated by scanning electron microscopy (SEM) and transmission electron microscopy (TEM). The SEM images (Figure 2a and Figure S2) show that the graphene sheets and CNTs are entangled forming a continuous all-carbon 3D network (Figure S3). TEM images (Figure $2 \mathrm{~b}$ and Figure $2 \mathrm{c}$ ) show that the CNTs are tightly attached to the few-layer graphene sheets. The nitrogen adsorption isotherm of a G-CNT100/20 (Figure 2d) is characterized by combined features, suggesting the hierarchical pore structure. The hybrid shows a specific surface area of $\sim 480 \mathrm{~m}^{2}$ $\mathrm{g}^{-1}$, calculated by the Brunauer-Emmett-Teller (BET) method. The high surface area and the hierarchical pore structure allow fast mass transfer and provide many accessible catalytic sites for $\mathrm{ORR}^{27,28}$.

The ORR kinetics performance of the G-CNT. Alkaline solution is the mostly used electrolyte for metal-air batteries ${ }^{1,2,29}$. Therefore, the ORR performance of our G-CNT hybrids were tested in a $0.1 \mathrm{M}$ $\mathrm{KOH}$ electrolyte. We combined cyclic voltammetry $(\mathrm{CV})$, rotating disk electrode (RDE) and rotating ring disk electrode (RRDE) measurements. A commercial $\mathrm{Pt} / \mathrm{C}$ catalyst was tested as a reference (20 wt. \% Pt, HiSPEC 3000 from Johnson Matthey Fuel Cells, Figure S4). In a $\mathrm{N}_{2}$-saturated electrolyte, redox features are negligible for both G-CNT100/20 and Pt/C (Figure 3a). While in an $\mathrm{O}_{2}$-saturated electrolyte, the G-CNT 100/20 exhibits a pronounced cathodic ORR peak at $-0.22 \mathrm{~V}$ versus an $\mathrm{Ag} / \mathrm{AgCl}$ reference electrode which is the same as shown by doped graphene $e^{16,17,30-32}$, although it is lower than that of $\mathrm{Pt} / \mathrm{C}$. It is worth noting that its peak current densityis about twice that for $\mathrm{Pt} / \mathrm{C}$, suggesting the high ORR activity of G-CNT 100/20. From the results shown in Figure $3 \mathrm{~b}$, it also shows a much higher ORR activity than a pure graphene monolith (Figure S5) and a physical blend of graphene and CNTs (Figure S6), revealing the uniqueness of the carefully engineered G-CNT hybrid. These results emphasize that a physical blend of graphene and CNTs could not develop an equivalent activity to that of the G-CNT 100/20 sample, and that the sample processing approach could sensibly alter the catalytic nature of the hybrids.

RDE measurements were used to reveal the ORR kinetics of GCNT100/20 and Pt/C. The linear-sweep voltammetry (LSV) curves of G-CNT100/20 at different rotation speeds are shown in Figure 3c. The onset potential and the half-wave potential of G-CNT100/20 are lower than those of $\mathrm{Pt} / \mathrm{C}$, but the limiting diffusion current is higher than that of $\mathrm{Pt} / \mathrm{C}$ in the range of $-0.5 \sim-0.8 \mathrm{~V}$ (Figure S7). According to the LSV curves at various rotation rates, the Koutecky-Levich plot at a potential of $-0.35 \mathrm{~V}$ is shown in the inset of Figure 3c. The electron transfer number is 3.86 from the slopes of Koutecky-Levich plots for G-CNT 100/20, very close to that of a commercial $\mathrm{Pt} / \mathrm{C}$ catalyst $(\mathrm{n}=3.94)$, and the variances of G-CNT $100 / 20$ and $\mathrm{Pt} / \mathrm{C}$ are $8.6 \times 10^{-4}$ and $6.2 \times 10^{-4}$, respectively. The dependence of electron transfer number $(\mathrm{n})$ on the CNT fraction is shown in Figure $3 \mathrm{~d}$. With the larger percentage of CNTs, the higher $\mathrm{n}$ number is obtained. This strongly indicates that CNTs function like a "dopant" to activate the catalytic nature of the pure graphene and the hybrids; or vice versa that graphene can "dope" CNTs to trigger the activity and adjust the selectivity. The as-obtained G-CNT hybrid exhibits a close to $4 \mathrm{e}$ selectivity for ORR; whereas both the pristine CNTs and graphene with metal impurities present a 2e ORR pathway (Table S1), which is reasonable as metal-nitrogen complexes are believed as the active sites for $4 \mathrm{e} \mathrm{ORR}^{5}$. It is also noted that small amount of residual metals does not enhance the ORR activity of the G-CNT hybrid. Therefore we could conclude that the trace amount metals determined in our undoped graphene or carbon nanotubes do not show the same "visible" electrocatalytic behavior as those observed in the metal-nitrogen complexes, or in the high amount conditions.

To further verify the ORR catalytic performance of G-CNT 100/ 20, RRDE measurement was conducted to monitor the amount of $\mathrm{H}_{2} \mathrm{O}_{2}$ generated at the disk electrode during the ORR process. The disk and ring currents were measured and are shown in Figure $3 \mathrm{e}$. The measured $\mathrm{H}_{2} \mathrm{O}_{2}$ yield is below $12 \%$ over the potential range of

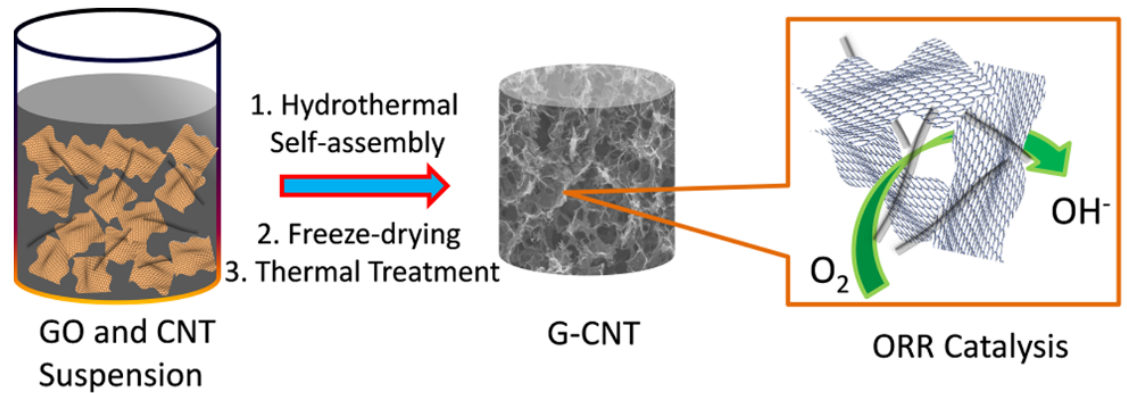

Figure 1 Scheme of fabrication process for the G-CNT material and its ORR catalysis. 

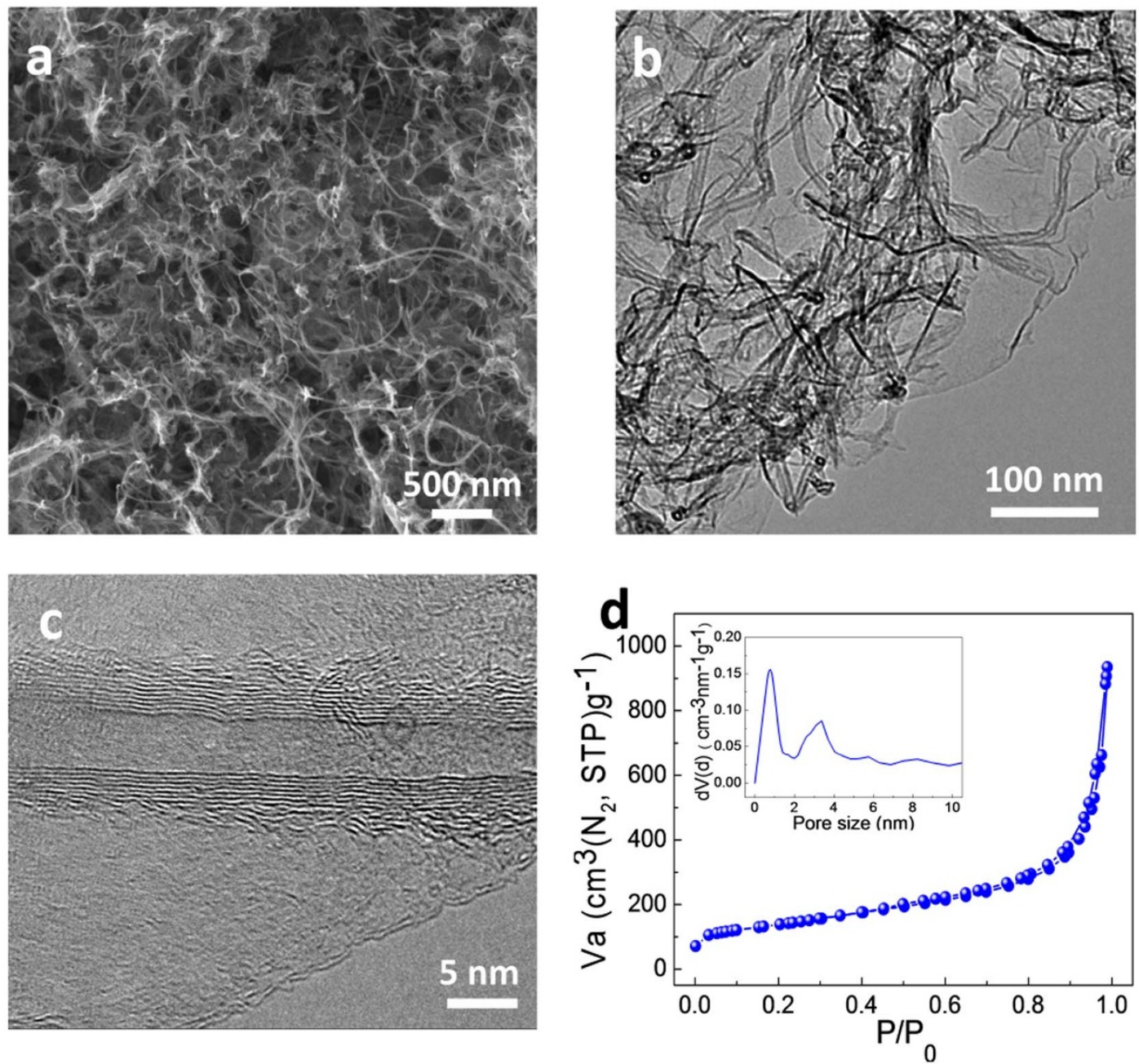

Figure $2 \mid$ Structure and morphology of G-CNT 100/20. (a) SEM image; (b) low magnification and (c) high magnification TEM images; (d) Nitrogen adsorption/desorption isotherm and pore size distribution (inset), demonstrating the hierarchical pore structure.

$-0.3 \sim-1.2 \mathrm{~V}$, giving an electron transfer number of $\sim 3.8$ consistent with Koutecky-Levich analysis (Figure $3 \mathrm{f}$ ). We tested the ORR performance of the all-carbon catalyst on a nickel foam in both aqueous (Figure $3 \mathrm{~g}$ ) and organic (Figure $3 \mathrm{~h}$ ) electrolytes. The linear relationships existed between the peak current and $v^{1 / 2}$ indicate that this stationary ORR reaction is a diffusion-controlled process (Figure S8 and S9). This all-carbon catalyst showed a satisfactory activity in a Lithium- $\mathrm{O}_{2}$ cell (Figure $\mathrm{S} 10$ ). It is noted that there are many macropores which can provide numerous gas diffusion channels, and they could continuously supply oxygen to the interior of the electrode when the G-CNT hybrid is used as a gas diffusion cathode in Lithium- $\mathrm{O}_{2}$ batteries (Figure S10). The ORR performance of our all-carbon G-CNT catalysts, covalently doped graphenes and graphene-supported metals or metal oxides are compared in Figure $3 i^{8,17,30,33-38}$. This comparison highlights the position of the G-CNT hybrids as a new kind of promising oxygen reduction catalysts (Table S1 and S2).

The cyclic stability and durability of G-CNT. Another main challenge of an ORR catalyst for its application is the cyclic stability and durability. We assessed the cyclic stability of G-CNT $100 / 20$ using the accelerated durability test protocol (Department of Energy, USA) by cycling the catalysts on a RDE at $50 \mathrm{mV} \mathrm{s}^{-1}$ for 8000 cycles in an $\mathrm{O}_{2}$-saturated $0.1 \mathrm{M} \mathrm{KOH}$ solution ${ }^{7}$. After each of the $1^{\text {st }}$, $1000^{\text {th }}, 3000^{\text {th }}, 5000^{\text {th }}$ and $8000^{\text {th }}$ cycles, LSV measurement was performed at $1600 \mathrm{rpm}$. Figure 4a shows the excellent cyclic stability of the G-CNT 100/20, indicating the superior stability compared with the $\mathrm{Pt} / \mathrm{C}$ reference shown in Figure $4 \mathrm{~b}$. We also conducted the $\mathrm{RDE}$ measurement in an electrolyte containing 0.5 M methanol to characterize the resistance G-CNT 100/20 and $\mathrm{Pt} / \mathrm{C}$ to methanol poisoning (Figure $4 \mathrm{c}$ and $\mathrm{d}$ ). The ORR activity of $\mathrm{Pt} / \mathrm{C}$ is dramatically reduced. In contrast, the G-CNT 100/20 exhibits excellent resistance to methanol poisoning with little activity loss.

\section{Discussion}

We demonstrated that the sample processing approach is key to the high oxygen reduction activity (Figure $3 \mathrm{~b}$ ). The uniform distribution during the ultrasonication process and high pressure in the hydrothermal process following by high-temperature annealing may help to develop the delicate contact and interaction between the CNTs and graphene sheets. This could result in non-covalent "doping" between the two carbon allotropes that renders a high ORR performance. We combined atomic force microscopy (AFM), Kelvin probe force microscope (KPFM), Raman spectroscopy and simulation to identify the interaction between graphene sheets and CNTs in the GCNT hybrid. A typical AFM image of a dispersed sample and the corresponding height profile are shown in Figure 5a and $5 \mathrm{c}$, which show a CNT overlapping with a graphene sheet. Figure $5 \mathrm{~b}$ shows the surface potential image of the marked area in Figure 5a, from which a higher surface potential along the direction of CNT is revealed. This result demonstrates that the modified local charge density distri- 

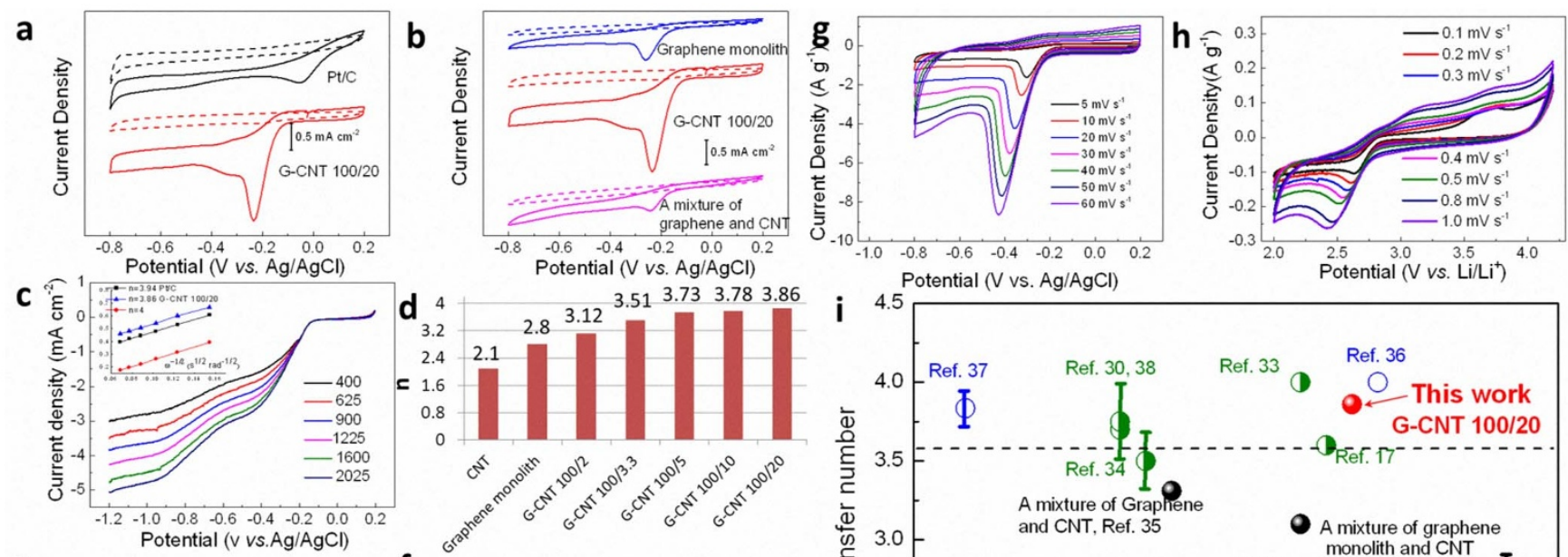

e
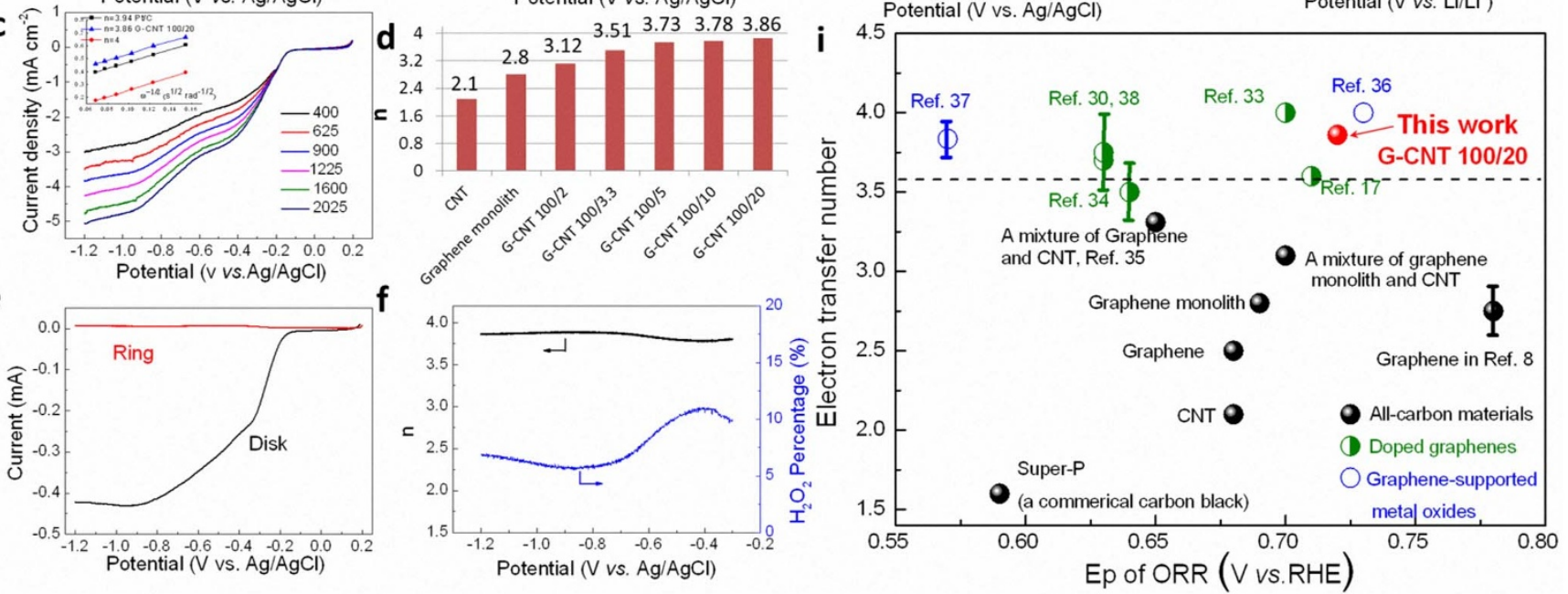

Figure 3 Performance of G-CNT 100/20 as an all-carbon catalyst. (a, b) CV curves of G-CNT 100/20, a commercial Pt/C catalyst and some reference samples including a graphene monolith and a simple mixture of graphene and $\mathrm{CNT}$ in $\mathrm{N}_{2}$-saturated (dashed line) and $\mathrm{O}_{2}$-saturated (solid line) electrolytes (Note that the loading density of catalyst materials is the same in these tests); (c) LSV curves of G-CNT 100/20 in $\mathrm{O}_{2}$-saturated electrolytes at different rotation rates (in rpm), The inset shows the corresponding Koutecky-Levich plot of G-CNT 100/20 and Pt/C derived from the RDE curves at $-0.35 \mathrm{~V}$; (d) Electron transfer number of CNT, graphene monolith and G-CNTs; (e) RRDE voltammogram of G-CNT 100/20 in an O ${ }_{2}$-saturated electrolyte; (f) Percentage of $\mathrm{H}_{2} \mathrm{O}_{2}$ and the electron transfer number of G-CNT 100/20 at various potentials, based on the corresponding RRDE data in e; The CV curves of catalyst loaded electrode of G-CNT 100/20 in $\mathrm{O}_{2}$-saturated $0.1 \mathrm{M} \mathrm{KOH}$ solution (g) and $1 \mathrm{M} \mathrm{LiCF}_{3} \mathrm{SO}_{3}$ in $\mathrm{TEGDME} \mathrm{h}$ ); (i) comparison of ORR performance for some typical all-carbon materials, doped graphenes and graphene-supported metals or metal oxides.

bution around the contact area. It is already known that CNTs can create a positively charged area on graphene sheets ${ }^{22}$. Therefore the positive potential should be of the top graphene sheet; this means the nanotube is beneath. Raman spectroscopy provides a sensitive probe for electronic structures of carbon materials including doped ones, and the doping-induced shift of the Raman-active tangential G-band results from the electronic structure changes (changes of Fermi level) ${ }^{39}$. The Raman spectra of G-CNT (Figures 5e and S11) show a stepwise downshift of the G-band as CNT fraction increases, which is usually caused by hole doping in graphene $e^{39,40}$. This agrees with the KPFM results.

The oxygen adsorption of G-CNTs was conducted to determine the enhanced oxygen adsorption capacity due to the non-covalent "doping" (Figure 5f). Figure 5g demonstrates that the larger fraction of CNTs induces the higher adsorption amount, which is consistent with the improved ORR activity (Figure 3d). Furthermore, the affinity of the all-carbon catalyst surface to oxygen was also studied by using differential scanning calorimetry (DSC) analysis, as shown in Figure $5 \mathrm{~h}$. The exothermal peak for G-CNT 100/20 is lower than the pure graphene monolith (without CNTs) and G-CNT $100 / 5$ by $81^{\circ} \mathrm{C}$ and $17^{\circ} \mathrm{C}$, respectively. This suggests the much easier chemisorption of oxygen on G-CNT 100/20 (oxidation of carbon atoms), which is in strong agreement with its higher adsorption capaicty for oxygen. These results confirm that the enhanced charge separation at the G-CNT interfaces is reponsible for the improved ORR activity. Our simulation illustrated the disturbed charge distribution in the G-CNT hybrid (see Supplementary Information). We noticed the positive charge $(0.232)$ on a graphene sheet in the hybrid compared to the zero charge on a graphene sheet without a CNT. This is evident of the hole doping from CNTs to graphene sheets ${ }^{25}$, and more fundamental simulation studies are ongoing. As increasing the amount of CNTs, the density of active sites increases, and hence the ORR activity becomes better, as demonstrated in Figure $3 \mathrm{~d}$.

We propose our understanding of the mechanism. KPFM results demonstrate the charge separation at the interface of the graphene and carbon nanotube (Figure 5). Due to the smaller work function of graphene than that of carbon nanotubes, the Fermi level of graphene is higher. According to the quantum tunneling theory, it is easier for the electrons in the graphene to tunnel through the vacuum barrier and jump into carbon nanotubes. The interfacial electron tunneling induced by the mismatch of the Fermi level on the planar and curved $s p^{2}$ surfaces eventually enables the charge separation at the interface (Figure 6). It is also shown that only particularly engineered interface can support such delicate electron tunnelling. The combined hydrothermal and high-temperature annealing treatment is responsible for the formation of such subtle interface between the two nanocarbon allotropes with different electronic characteristics ${ }^{19,25}$. The activated interface between graphene and CNT provides an ideal active place for enabling high ORR performance ${ }^{14,41-43}$. Very Recently, Cheon et al. have also used work function to understand the activity and reaction kinetics of ORR for heteroatom-doped carbons ${ }^{44}$, and in their case, a similar concept is proposed to what we used above to explain the unusual ORR activity of the all-carbon material, G-CNT hybrid.

In summary, we have combined hydrothermal and high-temperature annealing methods to engineer the interface between graphene and carbon nanotubes. The all-carbon product is highly active for oxygen reduction reaction; and the high activity/select- 

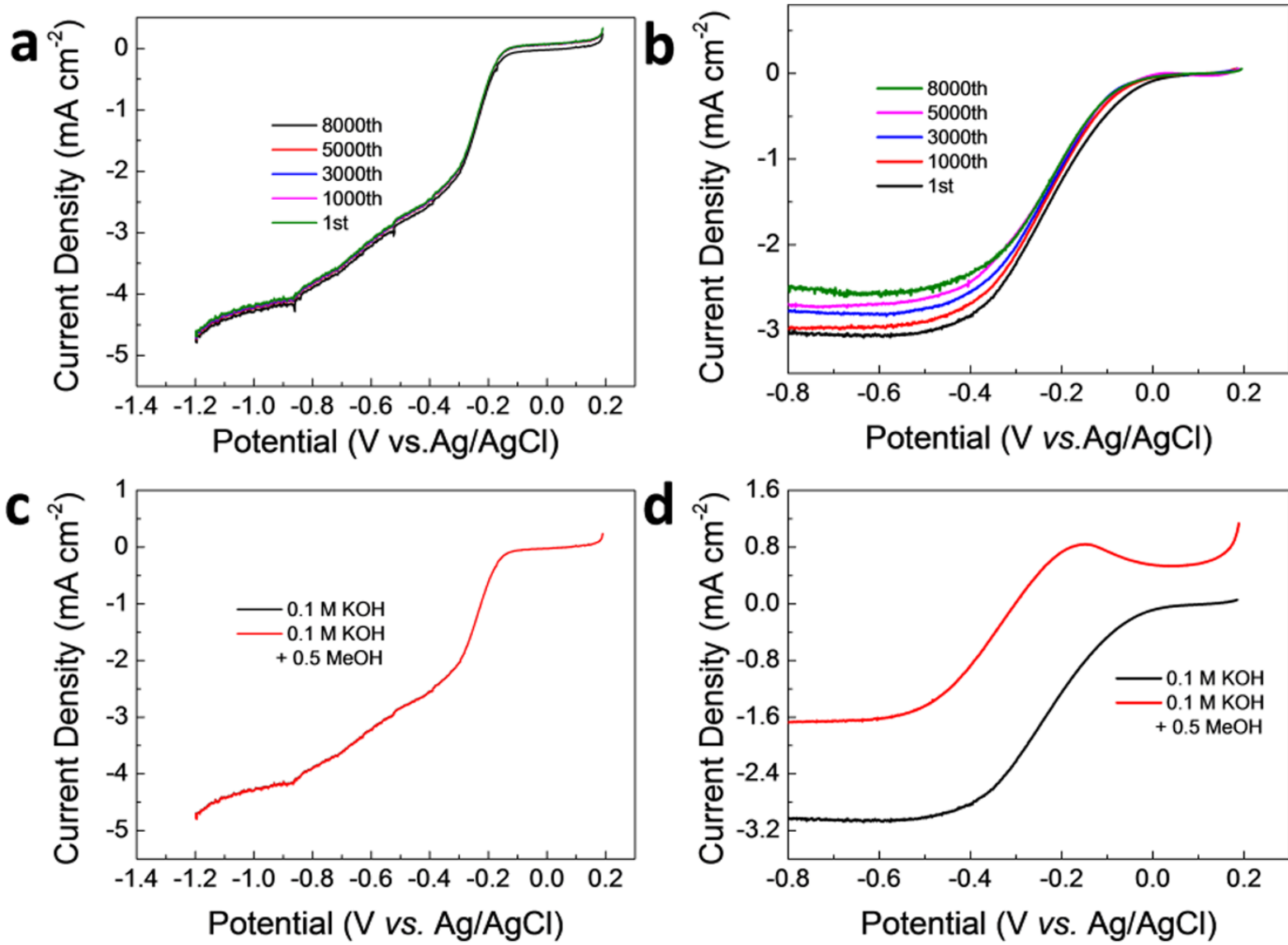

Figure $4 \mid$ RDE polarization curves of (a) G-CNT 100/20 and (b) Pt/C after the 1st, 1000th, 3000th, 5000th and 8000th in an $\mathrm{O}_{2}$-saturated 0.1 $\mathrm{M}$ $\mathrm{KOH}$ solution; RDE polarization curves of (c) G-CNT 100/20 and (d) $\mathrm{Pt} / \mathrm{C}$ in an $\mathrm{O}_{2}$-saturated $0.1 \mathrm{M} \mathrm{KOH}$ solution with or without $0.5 \mathrm{M}$ methanol. G-CNT 100/20 exhibits a dramatically improved tolerance to methanol poisoning compared with Pt/C.

ivity is triggered by the electron tunnelling and charge separation because of the distinct work functions between graphene and CNTs. Overall, the unique G-CNT hybrid shows comparable catalytic performance to a commercial $\mathrm{Pt} / \mathrm{C}$ catalyst and much higher cyclic stability and durability. More notably, the 3D open network of the all-carbon product is advantageous for gas diffusion and has shown promising potential as Lithium- $\mathrm{O}_{2}$ battery cathode catalysts.
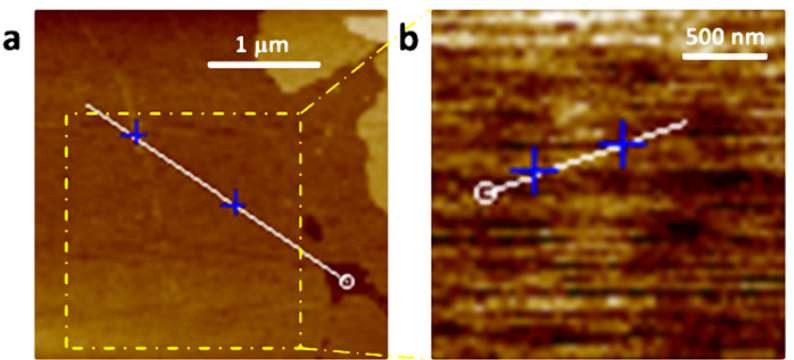

C
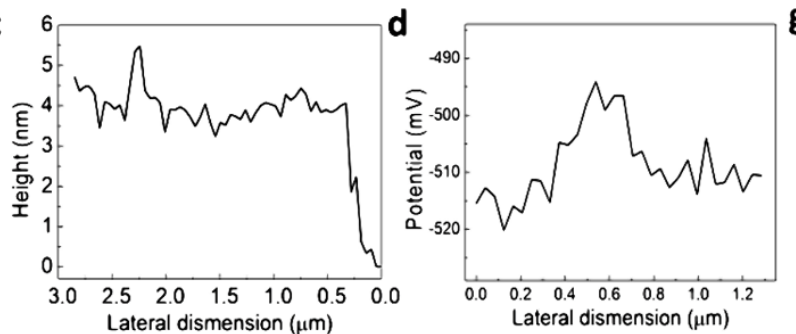
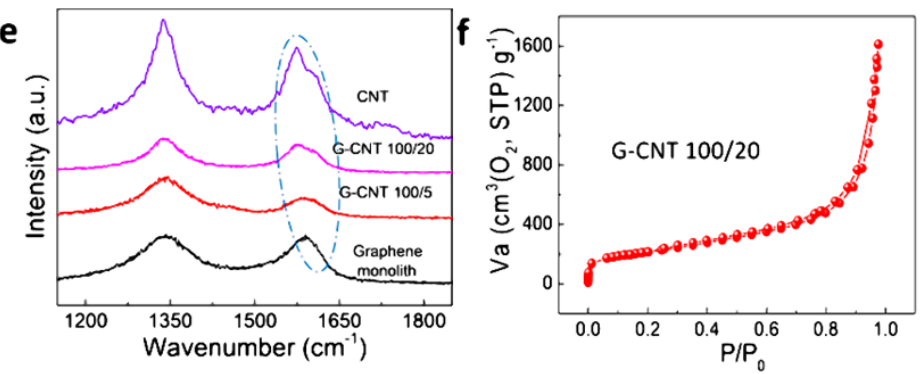

g

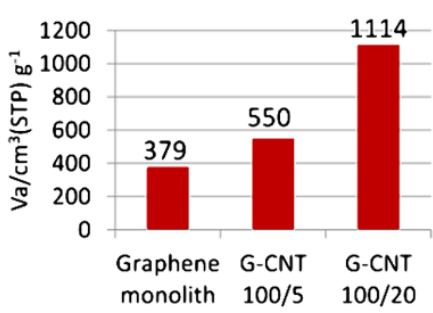

h

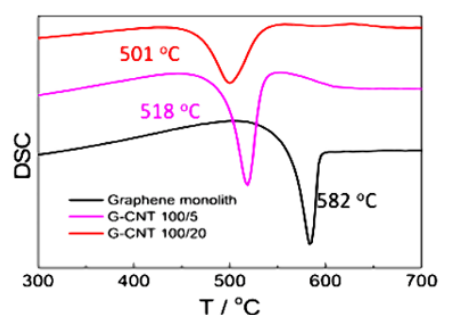

Figure 5 Evidence for the charge separation at the interface of the graphene and carbon nanotube. (a) AFM image and (c) corresponding height profile of G-CNT; (b) Surface potential image and (d) corresponding potential distribution of G-CNT; (e) Raman spectra of G-CNTs and some reference samples including CNT and graphene monolith; (f) Oxygen adsorption/desorption isotherm of G-CNT 100/20; (g) The oxygen adsorption amount at the relative pressure of 0.95 for G-CNTs; (h) DSC curves of G-CNT100/20, G-CNT100/5 and graphene monolith. 


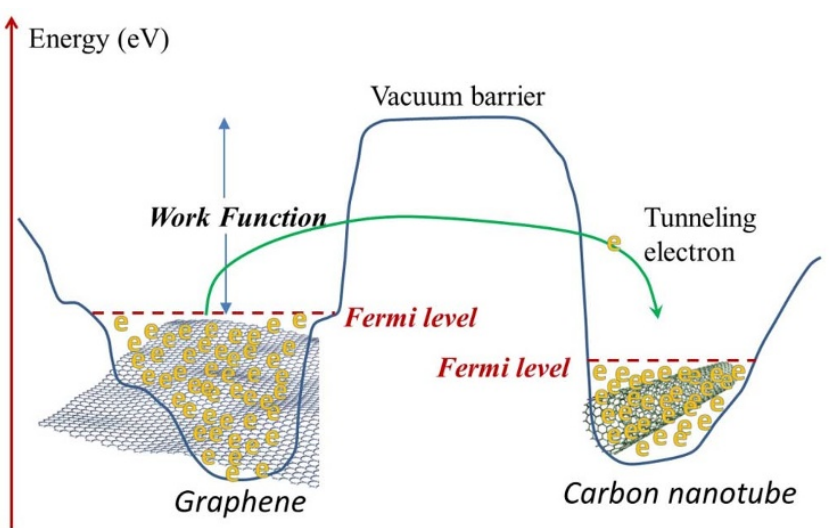

Figure $6 \mid$ Scheme of the charge separation at the interface between the graphene and carbon nanotube.

\section{Methods}

Preparation of G-CNT. Graphite oxide was prepared by oxidation of the graphite powder using a modified Hummers method. The CNT was prepared by the arcdischarge method without any catalysts. The homogeneous graphene oxide (GO) and CNT colloidal suspension was prepared by ultrasonication of graphite oxide $(170 \mathrm{mg})$ and CNTs in deionized water $(85 \mathrm{~mL})$ for $3 \mathrm{~h}$. The mass of CNTs is varied to obtain different mass ratios of $\mathrm{GO}$ and CNTs. Then the mixture was placed into a Teflon-lined autoclave $(100 \mathrm{~mL})$, and treated by the hydrothermal process at $150^{\circ} \mathrm{C}$ for $20 \mathrm{~h}$. After the autoclave was naturally cooled to the room temperature, a cylindrical hybrid hydrogel was obtained and then dried by a freeze-drying method. Finally, a G-CNT hybrid monolith was obtained by heating the as-prepared dry sample at $300^{\circ} \mathrm{C}$ for $2 \mathrm{~h}$ and $800^{\circ} \mathrm{C}$ for $3 \mathrm{~h}$ under an $\mathrm{Ar}$ atmosphere. The monolith was ground into powder for catalyst loading.

Preparation of graphene monolith. The graphene monolith was prepared by the hydrothermal treatment of graphene oxide suspension free of CNTs following the same condition as that for G-CNT.

Preparation of a simple mixture of graphene and CNT. Graphene was prepared by a vacuum-promoted exfoliation method ${ }^{45}$, and heated at $300^{\circ} \mathrm{C}$ for $2 \mathrm{~h}$ and $800^{\circ} \mathrm{C}$ for $3 \mathrm{~h}$ under an $\mathrm{Ar}$ atmosphere. CNTs were also annealed in the same condition. A simple mixture was prepared by mixing these pre-treated graphene and CNTs under strong sonication, which was then subjected to drying at $110^{\circ} \mathrm{C}$ for $10 \mathrm{~h}$ to form a dry powder.

Sample Characterizations. The morphology of the as-prepared samples was investigated by scanning (SEM, S4800, Hitachi) and transmission (TEM, 2100F, JEOL) electron microscopy. X-ray photoelectron spectroscopy (XPS) analysis was conducted with a Physical Electronics PHI5802 instrument using X-rays magnesium anode (monochromatic $\mathrm{K} \alpha \mathrm{X}$-rays at $1253.6 \mathrm{eV}$ ) as the source. Raman spectra were recorded using a multi-wavelength micro-Raman spectroscope (JY HR800) utilizing $532.05 \mathrm{~nm}$ incident radiation and a $50 \times$ aperture. Nitrogen adsorption experiments were conducted at $77 \mathrm{~K}$ using a BELsorp mini-instrument (BEL). Oxygen adsorption experiments were conducted at $77 \mathrm{~K}$ using an ASAP2020M (Micromeritics). Before the adsorption measurements, the samples were degassed in vacuum at $473 \mathrm{~K}$ for $12 \mathrm{~h}$. The specific surface areas of the samples were calculated by Brunauer-EmmettTeller (BET) analyses of their adsorption isotherms. Thermo gravimetric analysis and differential scanning calorimetry (NETZSCH STA 449 F3 Jupiter) measurements were performed from room temperature to $900^{\circ} \mathrm{C}$ with a heating rate of $5^{\circ} \mathrm{C} \mathrm{min}^{-1}$ in air. Atomic force microscopy (AFM) and Kelvin probe force microscope (KPFM) measurements were conducted for the sample which was sonicated to form a uniform dispersion and dropped onto a fresh silicon surface (peak force tapping mode, Bruke Multimode VIII).

1. Bruce, P. G., Freunberger, S. A., Hardwick, L. J. \& Tarascon, J. M. Li-O2 and Li-S batteries with high energy storage. Nat. Mater. 11, 19-29 (2012).

2. Cheng, F. \& Chen, J. Metal-air batteries: from oxygen reduction electrochemistry to cathode catalysts. Chem. Soc. Rev. 41, 2172-2192 (2012).

3. Wang, D.-W. \& Su, D. S. Heterogeneous nanocarbon materials for oxygen reduction reaction. EnergyEnviron. Sci. 7, 576-591 (2013).

4. Guo, S., Zhang, S. \& Sun, S. Tuning Nanoparticle Catalysis for the Oxygen Reduction Reaction. Angew. Chem. Int. Ed. 52, 8526-8544 (2013).

5. Bezerra, C. W. B. et al. A review of $\mathrm{Fe}-\mathrm{N} / \mathrm{C}$ and $\mathrm{Co}-\mathrm{N} / \mathrm{C}$ catalysts for the oxygen reduction reaction. Electrochim. Acta. 53, 4937-4951 (2008).

6. Lefèvre, M., Proietti, E., Jaouen, F. \& Dodelet, J-P. Iron-Based Catalysts with Improved Oxygen Reduction Activity in Polymer Electrolyte Fuel Cells. Science 324, 71-74 (2009).
7. Wu, G., More, K. L., Johnston, C. M. \& Zelenay, P. High-performance electrocatalysts for oxygen reduction derived from polyaniline, iron, and cobalt. Science 332, 443-447 (2011).

8. Liang, Y. et al. $\mathrm{Co} 3 \mathrm{O} 4$ nanocrystals on graphene as a synergistic catalyst for oxygen reduction reaction. Nat. Mater. 10, 780-786 (2011).

9. Yang, D.-S., Bhattacharjya, D., Inamdar, S., Park, J. \& Yu, J.-S. Phosphorus-Doped Ordered Mesoporous Carbons with Different Lengths as Efficient Metal-Free Electrocatalysts for Oxygen Reduction Reaction in Alkaline Media. J. Am. Chem. Soc. 134, 16127-16130 (2012).

10. Li, Q., Zhang, S., Dai, L. \& Li, L.-S. Nitrogen-Doped Colloidal Graphene Quantum Dots and Their Size-Dependent Electrocatalytic Activity for the Oxygen Reduction Reaction. J. Am. Chem. Soc. 134, 18932-18935 (2012).

11. Zhang, Y. et al. Manageable N-doped graphene for high performance oxygen reduction reaction. Sci. Rep. 3, 2771 (2013).

12. Liang, J., Du, X., Gibson, C., Du, X. W. \& Qiao, S. Z. N-Doped Graphene Natively Grown on Hierarchical Ordered Porous Carbon for Enhanced Oxygen Reduction. Adv. Mater. 25, 6226-6231 (2013).

13. Ding, W. et al. Space-Confinement-Induced Synthesis of Pyridinic-and PyrrolicNitrogen-Doped Graphene for the Catalysis of Oxygen Reduction. Angew. Chem. Int. Ed. 125, 11971-11975 (2013).

14. Gong, K., Du, F., Xia, Z., Durstock, M. \& Dai, L. Nitrogen-Doped Carbon Nanotube Arrays with High Electrocatalytic Activity for Oxygen Reduction. Science 323, 760-764 (2009).

15. Sheng, Z.-H., Gao, H.-L., Bao, W.-J., Wang, F.-B. \& Xia, X.-H. Synthesis of boron doped graphene for oxygen reduction reaction in fuel cells. J. Mater. Chem. 22, 390-395 (2012).

16. Yang, Z. et al. Sulfur-doped graphene as an efficient metal-free cathode catalyst for oxygen reduction. ACS Nano 6, 205-211 (2011).

17. Liang, J., Jiao, Y., Jaroniec, M. \& Qiao, S. Z. Sulfur and Nitrogen Dual-Doped Mesoporous Graphene Electrocatalyst for Oxygen Reduction with Synergistically Enhanced Performance. Angew. Chem. Int. Ed. 51, 11496-11500 (2012).

18. Yang, Q.-H. Dreams may come: from fullerene, carbon nanotube to graphene. New Carbon Mater. 26, 1-4 (2011).

19. Ando, T. The electronic properties of graphene and carbon nanotubes. NPG Asia Mater. 1, 17-21 (2009).

20. Khomyakov, P. A. et al. First-principles study of the interaction and charge transfer between graphene and metals. Phys. Rev. B 79, 195425 (2009).

21. Giovannetti, G. et al. Doping Graphene with Metal Contacts. Phys. Rev. Lett. 101, 026803 (2008).

22. Shi, Y. et al. Work function engineering of graphene electrode via chemical doping. ACS Nano 4, 2689-2694 (2010).

23. Shiraishi, M. \& Ata, M. Work function of carbon nanotubes. Carbon 39 1913-1917 (2001).

24. Yu, Y.-J. et al. Tuning the graphene work function by electric field effect. Nano Lett. 9, 3430-3434 (2009).

25. Robert, P. T. \& Danneau, R. Charge distribution of metallic single walled carbon nanotube-graphene junctions. New J. Phys. 16, 13019-13049 (2014).

26. Jeon, I.-Y. et al. Large-scale production of edge-selectively functionalized graphene nanoplatelets via ball milling and their use as metal-free electrocatalysts for oxygen reduction reaction. J. Am. Chem. Soc. 135, 1386-1393 (2012).

27. Parlett, C. M. A., Wilson, K. \& Lee, A. F. Hierarchical porous materials: catalytic applications. Chem. Soc. Rev. 42, 3876-3893 (2013).

28. Xiao, J. et al. Hierarchically Porous Graphene as a Lithium-Air Battery Electrode. Nano Lett. 11, 5071-5078 (2011).

29. Lee, J.-S. et al. Metal-Air Batteries with High Energy Density: Li-Air versus ZnAir. Adv. Energy Mater. 1, 34-50 (2011).

30. Lin, Z., Waller, G., Liu, Y., Liu, M. \& Wong, C. P. Facile Synthesis of NitrogenDoped Graphene via Pyrolysis of Graphene Oxide and Urea, and its Electrocatalytic Activity toward the Oxygen-Reduction Reaction. Adv. Energy Mater. 2, 884-888 (2012).

31. Qu, L., Liu, Y., Baek, J.-B. \& Dai, L. Nitrogen-doped graphene as efficient metalfree electrocatalyst for oxygen reduction in fuel cells. ACS Nano 4, 1321-1326 (2010).

32. Sun, Y., Li, C. \& Shi, G. Nanoporous nitrogen doped carbon modified graphene as electrocatalyst for oxygen reduction reaction. J. Mater. Chem. 22, 12810-12816 (2012).

33. Yang, S., Feng, X., Wang, X. \& Müllen, K. Graphene-Based Carbon Nitride Nanosheets as Efficient Metal-Free Electrocatalysts for Oxygen Reduction Reactions. Angew. Chem. Int. Ed. 50, 5339-5343 (2011).

34. Chen, P., Xiao, T.-Y., Qian, Y.-H., Li, S.-S. \& Yu, S.-H. A Nitrogen-Doped Graphene/Carbon Nanotube Nanocomposite with Synergistically Enhanced Electrochemical Activity. Adv. Mater. 25, 3192-3196 (2013).

35. Jin, Z. et al. Metal-free selenium doped carbon nanotube/graphene networks as a synergistically improved cathode catalyst for oxygen reduction reaction. Nanoscale 4, 6455-6460 (2012).

36. Wang, H., Liang, Y., Li, Y. \& Dai, H. Co1-xS-Graphene Hybrid: A HighPerformance Metal Chalcogenide Electrocatalyst for Oxygen Reduction. Angew. Chem. Int. Ed. 50, 10969-10972 (2011).

37. Wu, Z.-S. et al. 3D Nitrogen-Doped Graphene Aerogel-Supported Fe3O4 Nanoparticles as Efficient Eletrocatalysts for the Oxygen Reduction Reaction. J. Am. Chem. Soc. 134, 9082-9085 (2012). 
38. Zhao, Y. et al. A Versatile, Ultralight, Nitrogen-Doped Graphene Framework. Angew. Chem. Inter. Ed. 51, 11371-11375 (2012).

39. Yang, Q.-H. et al. Dual Raman Features of Double Coaxial Carbon Nanotubes with N-Doped and B-Doped Multiwalls. Nano Lett. 5, 2465-2469 (2005).

40. Panchakarla, L. et al. Synthesis, structure, and properties of boron-and nitrogendoped graphene. Adv. Mater. 21, 4726-4730 (2009).

41. Hu, X., Wu, Y., Li, H. \& Zhang, Z. Adsorption and Activation of O2 on NitrogenDoped Carbon Nanotubes. J. Phys. Chem. C 114, 9603-9607 (2010).

42. Wang, S. et al. Oxygen-enriched carbon material for catalyzing oxygen reduction towards hybrid electrolyte Li-air battery. I. Mater. Chem. 22, 21051-21056 (2012).

43. Ni, S., Li, Z. \& Yang, J. Oxygen molecule dissociation on carbon nanostructures with different types of nitrogen doping. Nanoscale 4, 1184-1189 (2012).

44. Cheon, J. Y. et al. Intrinsic Relationship between Enhanced Oxygen Reduction ReactionActivity and Nanoscale Work Function of Doped Carbons. J. Am. Chem. Soc. 136, 8875-8878 (2014).

45. Lv, W. et al. Low-Temperature Exfoliated Graphenes: Vacuum-Promoted Exfoliation and Electrochemical Energy Storage. ACS Nano 3, 3730-3736 (2009).

\section{Acknowledgments}

We appreciate the help from the National Supercomputing Center in Shenzhen and the support from National Basic Research Program of China (2014CB932403), National Science Foundation of China (Nos. 51232005, 51372167 and 51302146), NSF of Tianjin, China (No. 12JCZDJC27400) and Shenzhen Basic Research Project (Nos.

JC201104210152A and JCYJ20130402145002430). D.-W.W. appreciates the support from Australian Research Council Discovery Project Scheme (DP110100550). We also thank the financial support from Guangdong Province Innovation R\&D Team Plan (No. 2009010025)

\section{Author contributions}

Q.-H.Y., W.W., Y.T. and D.-W.W. were mainly responsible for preparing the manuscript with further inputs from other authors. All the authors discussed the results and contributed to the manuscript. Q.-H.Y. conceived the project and W.W., Y.T., W.L., D.-W. W. and Q.-H.Y. designed the experiments. W.W. and Y.T. carried out the materials synthesis, structural characterization and electrochemical measurements. F.-Y.S. and L.K. conducted the simulation of the results. Q.-H.Y., D.-W.W., W.W., Y.T., W.L., F.-Y.S., J.L., B.H.L. and F.Y.K. discussed the results. W.W., W.L. D.-W.W. and Q.-H.Y. wrote the initial manuscript which was approved by all the authors.

\section{Additional information}

Supplementary information accompanies this paper at http://www.nature.com/ scientificreports

Competing financial interests: The authors declare no competing financial interests.

How to cite this article: Wei, W. et al. Unusual High Oxygen Reduction Performance in All-Carbon Electrocatalysts. Sci. Rep. 4, 6289; DOI:10.1038/srep06289 (2014).

This work is licensed under a Creative Commons Attribution-NonCommercialNoDerivs 4.0 International License. The images or other third party material in this article are included in the article's Creative Commons license, unless indicated otherwise in the credit line; if the material is not included under the Creative Commons license, users will need to obtain permission from the license holder in order to reproduce the material. To view a copy of this license, visit http:// creativecommons.org/licenses/by-nc-nd/4.0/ 